\title{
LY6K/VEGFR1/VEGFR2 Multipeptide Vaccine
}

National Cancer Institute

\section{Source}

National Cancer Institute. LY6KIVEGFR1/VEGFR2 Multipeptide Vaccine. NCI Thesaurus.

Code C74056.

A multipeptide vaccine consisting of peptides derived from lymphocyte antigen 6 complex locus K (LY6K) and type I and II vascular endothelial growth factor receptors (VEGFRs) with potential antineoplastic activity. Upon administration, LY6K/VEGFR1/VEGFR2 multipeptide vaccine may elicit an antitumor cytotoxic Tlymphocyte (CTL) immune response against LY6K-expressing tumor cells and/or VEGFRexpressing vascular endothelial cells involved in tumor angiogenesis. LY6K is a tumorassociated antigen (TAA) that occurs singly in glycosylphosphatidyl-inositol (GPI)-linked cell-surface glycoproteins or as three-fold repeated domain in the urokinase-type plasminogen activator receptor; VEGFRs are cell surface receptors that stimulate endothelial cell proliferation, invasion, angiogenesis, and vasculogenesis upon ligand binding and receptor activation. 